\section{Advances in risk assessment and prophylaxis for central nervous system relapse in diffuse large B-cell lymphoma}

\author{
David Qualls and Jeremy S. Abramson
}

Center for Lymphoma, Massachusetts General Hospital Cancer Center, Boston, MA, USA

\section{ABSTRACT}

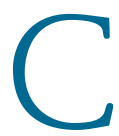

entral nervous system recurrence of diffuse large B-cell lymphoma is an uncommon but devastating event, making identification of patients at high risk for relapse within the central nervous system essential for clinicians. Modern risk stratification includes both clinical and biological features. A validated clinical risk model employing the five traditional International Prognostic Index risk factors plus renal or adrenal involvement can identify a high-risk patient population with a central nervous system recurrence risk of greater than 10\%. Lymphoma involvement of certain discrete extranodal sites such as the testis also confers increased risk, even in stage I disease. Adverse biological risk factors for central nervous system relapse include presence of translocations of $M Y C, B C L 2$ and/or BCL6, in so-called double- or triple-hit lymphoma. Immunohistochemically detectable co-expression of MYC and BCL2 in the absence of translocations also portends an increased risk of relapse within the central nervous system, particularly in the setting of the activated B-cell-like subtype of diffuse large B-cell lymphoma. The role, method, and timing of prophylactic therapy remain controversial based on the available data. We review both intrathecal and systemic strategies for prophylaxis in high-risk patients. Our preference is for systemic methotrexate in concert with standard chemoimmunotherapy in the majority of cases. Several novel agents have also demonstrated clinical activity in primary and secondary central nervous system lymphoma and warrant future investigation in the prophylactic setting.

\section{Introduction}

Diffuse large B-cell lymphoma (DLBCL) is the most common adult non-Hodgkin lymphoma, accounting for approximately one-third of all newly diagnosed cases in the United States. ${ }^{1,2}$ The prognosis has improved substantially since the introduction of rituximab nearly 20 years ago, with 5 -year overall survival rates of approximately $70 \%$ depending on the baseline characteristics of the patients and their disease..$^{3.5}$

Despite improvements in outcomes, a minority of patients with DLBCL will still suffer relapse within the central nervous system (CNS), which carries nearly universally poor outcomes with a median survival following diagnosis of CNS involvement of only 2-5 months. ${ }^{6-10}$ Indeed, secondary CNS lymphoma has long represented a great unmet medical need within the field of oncology, a need that has been particularly difficult to address since CNS involvement is usually an exclusion criterion for participation in clinical trials of novel agents. Given the significant morbidity and mortality associated with this event, much attention has been devoted to the identification of high-risk patients, and evaluation of therapies to mitigate the risk of CNS recurrence.

Another important area of investigation involves the diagnosis and treatment of occult leptomeningeal disease: lymphoma detected by cerebrospinal fluid (CSF) cytology or flow cytometry, without overt clinical signs or symptoms of CNS involvement by lymphoma. Occult disease has been shown to be significantly asso-
Haematologica 2019

Volume 104(1):25-34

\section{Correspondence:}

jabramson@mgh.harvard.edu

Received: July 25, 2018.

Accepted: November 15, 2018.

Pre-published: December 20, 2018.

doi:10.3324/haematol.2018.195834

Check the online version for the most updated information on this article, online supplements, and information on authorship \& disclosures: www.haematologica.org/content/104/1/25

(C)2019 Ferrata Storti Foundation

Material published in Haematologica is covered by copyright. All rights are reserved to the Ferrata Storti Foundation. Use of published material is allowed under the following terms and conditions:

https://creativecommons.org/licenses/by-nc/4.0/legalcode. Copies of published material are allowed for personal or internal use. Sharing published material for non-commercial purposes is subject to the following conditions:

https://creativecommons.org/licenses/by-nc/4.0/legalcode, sect. 3. Reproducing and sharing published material for commercial purposes is not allowed without permission in writing from the publisher. 
ciated with CNS relapse risk, as well as mortality. ${ }^{11-15}$ Few data are available to inform the optimal intensity of therapy required to eradicate occult CNS disease, but our practice is to treat occult CNS involvement by lymphoma in the same way as we treat active secondary CNS lymphoma.

The rate and patterns of CNS involvement with DLBCL have evolved with the introduction of rituximab-containing therapy. Most studies have found a slight decrease in the incidence of CNS relapse with rituximab use, with modern rates of $2-4 \% .^{7,16-19}$ This decrease is likely due to superior control of systemic disease, as well as a benefit from minimal penetration of the rituximab antibody in the CSF., ${ }^{7,20}$ Accordingly, localization of CNS relapse in the modern era has shifted to the brain parenchyma in the majority of cases, whereas relapse in the leptomeningeal compartment predominated prior to the introduction of rituximab.,20-21 Relapses within the CNS generally occur early in the treatment course, often presenting prior to completion of initial therapy or shortly thereafter. ${ }^{16,19}$

A significant proportion (14-48\%) of patients with CNS relapse also have systemic relapse at the time of diagnosis. ${ }^{11,12,22,23}$ While isolated CNS relapse may be prevented with effective CNS prophylaxis, concomitant systemic and CNS relapse likely represents a failure of systemic treatment, ${ }^{7,16}$ and patients with concurrent CNS and systemic relapse appear to have a worse prognosis than those with CNS relapse alone. ${ }^{24}$ Current studies do not reliably differentiate between isolated and concomitant systemic/CNS relapse when exploring the efficacy of CNS prophylaxis, so this remains an important area for future investigation.

CNS-directed prophylactic therapy has been widely utilized in DLBCL. This practice is based largely on the established benefit in other high-grade lymphomas with a high risk of CNS involvement, particularly Burkitt lymphoma and acute lymphoblastic leukemia. ${ }^{25,26}$ Data demonstrating a benefit of prophylactic strategies in DLBCL, however, have been limited and occasionally conflicting. This is largely due to the low overall risk of CNS events, as well as reliance on retrospective analyses and underpowered subset analyses of prospective studies, which suffer from small sample sizes and significant heterogeneity in indications and methods for prophylaxis. As a result, selection of appropriate patients for CNS prophylaxis, as well as the type and timing of prophylactic strategies, remain highly controversial.

In this review, we attempt to consolidate current information and examine the most recent advances regarding CNS risk assessment and approaches to CNS prophylaxis in patients with DLBCL.

\section{Evaluating risk of central nervous system recurrence}

Optimal CNS prophylaxis in DLBCL relies first upon accurately identifying the small proportion of high-risk patients who should be targeted for CNS evaluation and intervention. Modern risk stratification includes both traditional clinical and laboratory assessments, as well as incorporation of pathological and molecular characteristics of the patient's disease.

\section{Clinical risk models}

The risk of CNS involvement in patients with DLBCL is concentrated in high-risk populations with certain patientand disease-specific characteristics. Studies performed in the early 2000s demonstrated that patients with high International Prognostic Index (IPI) scores were at greater risk of CNS involvement. ${ }^{10,27-29}$ The original IPI consisted of five risk factors: age $>60$ years, elevated lactate dehydrogenase, Eastern Cooperative Oncology Group (ECOG) Performance Status $>1$, advanced stage disease, and involvement of more than one extranodal site. More recently, the German High Grade NHL Study Group (DSHNHL) and British Columbia Cancer Agency (BCCA) developed and validated the CNS International Prognostic Index (CNS-IPI) as a risk stratification tool to predict risk of CNS recurrence. ${ }^{18}$ They examined an initial cohort consisting of 2164 patients enrolled in DSHNHL clinical trials and identified five risk factors for CNS disease based on multivariate analysis: these include four of the five original IPI risk factors (with the exception of $>1$ extranodal site), plus either kidney or adrenal involvement. The presence of multiple extranodal sites was likely not a significant risk factor in multivariable analysis because of the overlap with advanced stage, but it was nonetheless retained in the final six-factor model for ease of application. Patients are stratified as having low (0-1 points), intermediate (2-3 points), or high (4-6 points) risk disease, which predicted 2 -year rates of CNS relapse of $0.8 \%, 3.9 \%$, and $12 \%$, respectively (Figure 1). The model was validated on 1597 patients in the BCCA database. Notably, additional risk factors for CNS recurrence were identified in the validation cohort based on multivariable analysis, which likely reflect differences in the populations of patients between the two cohorts; additional risk factors included $>1$ extranodal sites, and involvement of the testis, pericardium, orbit, or bone marrow.

Subsequent studies have confirmed the utility of the CNS-IPI, particularly in validating risk associated with the highest risk cohort with four to six risk factors. ${ }^{17,19,30}$

\section{High-risk extranodal sites}

Certain extranodal sites have been implicated as discrete risk factors for subsequent CNS relapse, though few have been found to be independently predictive of CNS involvement on multivariable analyses. Testicular involvement in DLBCL is the most well-established, with studies demonstrating CNS relapse rates of $12-25 \%$, even in stage I completely resected disease. ${ }^{31-34}$ Unlike other systemic DLBCL which typically recur early, primary testicular DLBCL can relapse in the CNS as late as 10 years after initial diagnosis, and occurs most commonly within the brain parenchyma. ${ }^{31,32}$ There appears to be a pathophysiological relationship between primary testicular DLBCL and primary CNS DLBCL. Both diseases tend to be activated $B$-cell $(A B C)$-like by transcriptional profiling, and share genetic features including oncogenic toll-like receptor signaling based on MYD88 mutations or NFKBIZ amplification, B-cell receptor pathway activation, and BCL-6 deregulation..$^{35}$ Altered adhesion molecule expression also likely contributes to the predilection of primary testicular DLBCL for immune privileged sites such as the testis and CNS, while the loss of HLA-DR and surface immunoglobulin and increased expression of PD-L1 and PD-L2 via 9p24.1 amplification contribute to immune escape. . $^{35,36}$

Additional sites of extranodal involvement have historically been associated with greater risk of CNS relapse, although on multivariate analysis these have not been consistently predictive in the modern era. These sites 
include the bone marrow, paranasal sinus, orbit, pericardium, ovary, uterus, and breast. ${ }^{37-41}$ Involvement of paranasal sinuses was identified as a significant risk in the pre-rituximab era, but recent data have demonstrated that the risk of CNS events is not increased in the era of rituximabbased therapy, and there is not a clear role for either intrathecal prophylaxis or consolidative radiation therapy in these patients. ${ }^{42}$ Kidney and adrenal involvement had previously been identified as high-risk locations, and are now included in the CNS-IPI, as previously discussed. ${ }^{43,44}$

\section{Biological risk factors}

As our understanding of the molecular pathogenesis of DLBCL has advanced, specific biological features have become increasingly important predictors of CNS risk. DLBCL harboring a MYC rearrangement has been associated with an increased risk of CNS recurrence and an inferior overall survival relative to other forms of DLBCL. ${ }^{45,46} \mathrm{~A}$ $M Y C$ rearrangement rarely occurs as a sole genetic abnormality, however, and the dominant prognostic impact appears to be conferred by the rearrangement in concert with additional genetic aberrations. ${ }^{45,46}$ High-grade B-cell lymphoma with translocations of MYC and BCL2 and/or $B C L 6$, also known as double- or triple-hit lymphoma, represents about $5 \%$ of all newly diagnosed large B-cell lymphomas and carries a poor prognosis with a median overall survival of less than 2 years. ${ }^{47,48} \mathrm{CNS}$ involvement is common at either diagnosis or relapse, and has been reported in as many as $50 \%$ of affected patients. ${ }^{48.50}$ Compared to routine DLBCL therapy, more aggressive initial chemoimmunotherapy regimens such as dose-adjusted EPOCH-R (etoposide, prednisolone, vincristine, cyclophosphamide, doxorubicin plus rituximab) are typically employed, along with intrathecal CNS prophylaxis. This is based primarily on retrospective analyses which have demonstrated improved progression-free survival with more aggressive induction regimens and have suggested improved survival for patients receiving intrathecal therapy in this disease in which CNS recurrences commonly involve the leptomeningeal compartment. ${ }^{47}$

Another area of interest has been DLBCL with immunohistochemically detectable expression of MYC and BCL2 without associated translocations, otherwise called double-expressing lymphoma. Dual protein expression is significantly more common than double-hit lymphoma, occurring in approximately $30 \%$ of cases of DLBCL. ${ }^{51,52} \mathrm{~A}$ retrospective analysis of double-expressing lymphoma by the BCCA found a CNS recurrence risk of approximately $9 \%$, compared to only $2 \%$ in non-double-expressing cases of DLBCL. ${ }^{51}$ This risk was modified, however, based on cell of origin, and by risk stratification according to the CNS-IPI score. The risk of CNS events in double-expressing lymphoma appears limited to the ABC-like subset of DLBCL in which the CNS relapse risk is approximately $15 \%$, while there is no apparent increased risk in doubleexpressing germinal center B-cell (GCB)-like disease. Risk also appears confined to the intermediate- and high-risk CNS-IPI patients in whom the CNS relapse rate approximates $12 \%$ and $22 \%$, respectively, without any increased risk among low-risk CNS-IPI patients with doubleexpressing lymphoma. These analyses reinforce the complexity of assigning risk of CNS relapse in DLBCL, which warrants attention to clinical, histopathological, and molecular factors for optimal risk estimation.

Novel methods for risk stratification are being devel- oped. The use of pretreatment positron emission tomography to predict CNS relapse has been proposed, with elevated total lesion glycolysis found to be predictive of an increased risk of CNS relapse on multivariable analysis. ${ }^{53}$ Additional biomarkers emerging from pathological analyses have been utilized to stratify risk, including ITGA10, CXCR5 and nuclear PTEN. ${ }^{54,55}$ Further research in these areas in concert with existing biomarkers and clinical risk stratification tools should be performed to determine the clinical utility of these studies before they are incorporated into routine clinical care.

\section{Baseline central nervous system evaluation}

CNS recurrence of DLBCL typically occurs early in the disease course, either during systemic treatment or within several months of completing treatment. 8,28 This suggests that subclinical involvement of the CNS by DLBCL is likely present at the time of diagnosis in such cases. Early identification of patients with CNS involvement is crucial, as the treatment and prognosis may be significantly altered based on this knowledge. As such, evaluation of the CNS via CSF analysis and/or neuroimaging should be considered in patients with high-risk features or neurological symptoms.

CSF analysis consists of conventional cytology and flow cytometry. Cytology alone has low sensitivity for CNS disease at less than $60 \%$ for leptomeningeal disease and virtually no ability to detect parenchymal lymphoma. ${ }^{56}$ The addition of flow cytometry significantly increases sensitivity for detection of occult CNS involvement, which can be found in approximately $10 \%$ of high-risk patients and is associated with a high rate of subsequent CNS progression and poor overall survival. ${ }^{11}$ ${ }^{15}$ An important caveat is that these data include patients predominantly treated in the pre-rituximab era, at a time when most CNS relapses involved the leptomeningeal compartment. ${ }^{28,57,58}$ Since the introduction of rituximabbased chemoimmunotherapy, the incidence of CNS

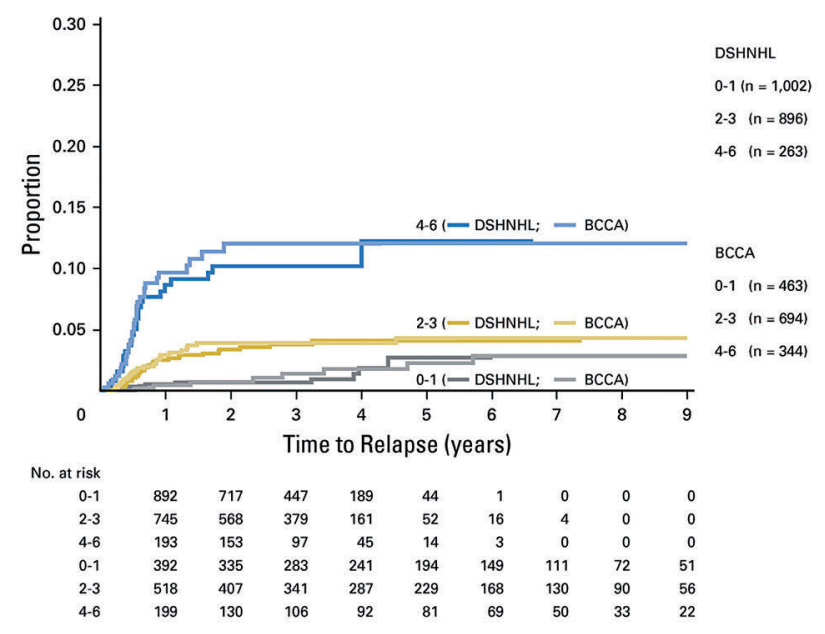

() 2016 by American Society of Clinical Oncology

Figure 1. Kaplan-Meier curve depicting risk of central nervous system relapse based on the Central Nervous System International Prognostic Index score. DSHNHL: German High Grade Lymphoma Study Group cohort, BCCA: British Columbia Cancer Agency cohort. Schmitz, et al. J Clin Oncol 2016. ${ }^{18}$ 
relapses has decreased and these relapses more commonly involve the brain parenchyma where CSF evaluation provides a lower diagnostic yield. ${ }^{7,20,21}$ As such, routine evaluation of the CSF in patients without neurological symptoms in the modern era remains controversial. Our own practice is to evaluate the CSF at baseline in all patients with neurological symptoms, in patients with disease infiltrating neural foramina, and in most patients with double- or triple-hit lymphoma, as these patients are at particularly high risk and often relapse within the leptomeninges. For high-risk asymptomatic DLBCL patients without double- or triple-hit cytogenetics who we are treating with intrathecal methotrexate for CNS prophylaxis, we evaluate their baseline CSF at the time of their initial intrathecal injection. For high-risk patients receiving CNS prophylaxis with high-dose systemic methotrexate, we consider whether a finding of an occult positive CSF would alter the patient's treatment plan as CNS-directed therapy is already planned. Patients with relative contraindications to CNS prophylaxis, or less clear indications for prophylaxis in whom the risk/benefit ratio is not as well defined, may benefit from CSF analysis to assist in clinical decision-making.

An alternative approach is to perform baseline CNS evaluation with CSF cytology and flow cytometry in all patients considered at high-risk of CNS relapse. Our practice has shifted away from this strategy in all highrisk patients as the rate of leptomeningeal relapse has declined in the rituximab era, decreasing the yield of broadly applied CSF testing. Furthermore, it remains unclear whether additional CNS-directed therapy for occult disease is needed if these patients are already receiving empiric systemic high-dose methotrexate as CNS prophylaxis. For patients receiving intrathecal rather than systemic CNS-directed chemotherapy, however, the finding of occult lymphoma within the CSF should prompt intensification of the intrathecal treatment regimen beyond what would be administered for prophylaxis alone. Ultimately the decision of whether to perform baseline CSF evaluation should be personalized to the patient based on that person's discrete CNS risk factors, symptomatology, medical comorbidities, and treatment plan.

One potential area for future research is the use of polymerase chain reaction to evaluate the presence of occult CNS disease. Recent studies in primary CNS lymphoma have shown that assessments for micro-RNA (miRNA) and U2 small nuclear RNA fragments (RNU21f) via polymerase chain reaction were able to detect primary CNS DLBCL with high sensitivity and specificity. ${ }^{59,60}$ Studies performed in other cancers with CNS involvement have also demonstrated the utility of nextgeneration sequencing of CSF cell-free DNA in detecting and characterizing CNS disease. ${ }^{61,62}$ While similar studies still need to be performed in secondary CNS lymphoma, it is possible that utilizing these techniques could further improve our ability to assess occult CNS involvement.

At initial diagnosis, a careful history and neurological examination should be performed in all patients. The presence of any neurological signs or symptoms warrants magnetic resonance imaging evaluation of the brain and/or spine based on the relevant clinical finding. In the absence of neurological signs or symptoms of concern, there are insufficient data to recommend routine baseline neuroimaging.

\section{Central nervous system prophylaxis}

Despite the widespread use of CNS prophylaxis in patients determined to be at high risk of CNS recurrence, its efficacy remains controversial. There are no randomized controlled trials designed specifically to determine whether prophylactic strategies reduce CNS events. Most relevant data, therefore, come from subset analyses of clinical trials which are not powered to determine the impact of CNS prophylaxis, and from retrospective analyses that are susceptible to selection and reporting biases. ${ }^{63}$ Interpretation of these data is further confounded by significantly varying protocols with different indications, timing, dosing, and chemotherapeutic agents employed for prophylaxis.

\section{Indications for central nervous system prophylaxis}

Ultimately the goal of CNS prophylaxis is to minimize the incidence of CNS relapse, while allocating such therapy to those at highest risk and sparing those with low-risk disease unnecessary toxicity. As discussed above, there is a spectrum of risk associated with specific disease features and patients' characteristics, and the threshold for use of prophylaxis varies from clinician to clinician.

Based on the aforementioned discussion of biological and clinical risk stratification, prophylactic CNS-directed therapy should be considered for nearly all patients with double- or triple-hit lymphoma, DLBCL patients with a high-risk CNS-IPI score (4-6 risk factors), and intermediate-risk patients (2-3 risk factors) who are $\mathrm{ABC}$-subtype with dual expression of MYC and BCL2. We also recommend CNS prophylaxis in patients with disease in selected high-risk anatomic locations, including primary testicular DLBCL, orbital disease involving the globe or posterior compartment, and disease directly infiltrating spinal neuroforamina. CNS prophylaxis in patients with multiple other discrete extranodal locations remains more controversial based on available data and should be personalized in the context of the overall clinical and biological risk factors for the patient.

\section{Intrathecal therapy}

Intrathecal chemotherapy, particularly methotrexate, has been the most widely employed method of prophylaxis. Despite extensive data available, however, a protective benefit favoring this approach for prevention of CNS relapse has never been established, either before or after the introduction of rituximab.

Three prospective studies in the rituximab era found no benefit from intrathecal methotrexate among patients defined as high risk. The RICOVER- 60 trial conducted by the DSHNHL compared CHOP-14 (cyclophosphamide, doxorubicin, vincristine and prednisolone at 14-day intervals) to R-CHOP-14 (CHOP-14 plus rituximab) in patients over 60 years of age and recommended CNS prophylaxis with intrathecal methotrexate in patients with involvement of the testes, head or upper neck. Among 1222 subjects, 273 received at least one cycle of intrathecal methotrexate. Notably, only $57 \%$ of patients on this trial who met criteria for CNS prophylaxis actually received it, perhaps reflecting a lack of enthusiasm for this approach by treating investigators. When comparing CNS recurrence rates within this targeted population based on administration of prophylaxis, no significant preventive benefit could be identified. ${ }^{16}$ These findings were replicat- 
ed in a broader analysis of 2210 patients treated on additional prospective clinical trials by the DSHNHL, 620 of whom received rituximab-based treatment: intrathecal methotrexate again yielded no reduction in risk of CNS events. $^{64}$

Similarly, a randomized controlled trial comparing RCHOP-14 versus R-CHOP-21 (cyclophosphamide, doxorubicin, vincristine and prednisolone plus rituximab at 21day intervals) included 984 patients, of whom 177 received CNS prophylaxis with the vast majority (92\%) receiving intrathecal methotrexate. When stratified based on CNS-IPI, patients treated with intrathecal methotrexate had similar rates of CNS relapse, progression-free and overall survival compared with those not given prophylaxis. ${ }^{19}$ Several additional retrospective analyses involving large numbers of patients treated with rituximab-based chemoimmunotherapy in the modern era have likewise failed to demonstrate an association between intrathecal methotrexate use and reduction in CNS relapse rates. ${ }^{22,65-67}$

The lack of clinical benefit observed with intrathecal methotrexate may be explained by the pharmacokinetics of this drug. Historic experiments found that methotrexate concentrations within the neuroaxis varied widely between different patients when the drug was administered via lumbar puncture. In one study, two of nine patients given intrathecal methotrexate did not meet the target therapeutic concentration at any time, and five of the nine did not sustain therapeutic concentrations for 24 hours. ${ }^{68}$ Another study monitoring the distribution of radionuclide Indium showed that it could take up to 24 hours for intrathecal injections of Indium to appear in the ventricles, suggesting that intrathecal therapy injected at the lumbar sac may fail to protect the cerebral leptomeninges due to uneven distribution. ${ }^{69}$ Furthermore, intrathecal methotrexate fails to achieve therapeutic concentrations within the brain parenchyma, ${ }^{70}$ which could lead to reduced efficacy in this site where the majority of CNS relapses occur in the rituximab era. ${ }^{6,7,20,21}$

It is important to note the significant limitations of studies evaluating the efficacy of intrathecal methotrexate. While available data including non-randomized prospective and retrospective studies have not reliably demonstrated lower CNS relapse rates in patients receiving intrathecal therapy, these data are heterogeneous and cannot be considered definitive in their conclusions. Only appropriately powered randomized trials can truly exclude the possibility that intrathecal methotrexate reduces the risk of CNS relapse, although such trials would be extremely difficult to conduct. In cases in which alternative therapies, such as high-dose intravenous methotrexate, cannot be administered, intrathecal methotrexate remains a reasonable option.

One scenario in which intrathecal methotrexate remains an appropriate standard therapy is when administered with the dose-adjusted EPOCH-R regimen in patients with Burkitt lymphoma or high grade B-cell lymphoma (including double-hit and triple-hit lymphoma), in which it has been the exclusively studied method of CNS protection. Intrathecal methotrexate has been demonstrated to improve the clinical outcome in these histological types of lymphoma which frequently relapse in the CSF., ${ }^{47,71}$

\section{Systemic chemotherapy}

Effective systemic therapy which crosses the bloodbrain barrier may overcome the liabilities of intrathecal therapy and achieve even and predictable concentrations throughout the entire neuroaxis, including both the leptomeningeal and parenchymal compartments. ${ }^{72}$

Consideration of systemic CNS prophylaxis is derived largely from experience in primary CNS DLBCL, in which high-dose systemic methotrexate improves progressionfree and overall survival and remains the standard backbone of first-line treatment. ${ }^{73,74}$ The efficacy of systemic methotrexate as prophylactic therapy for the CNS has also been validated in acute lymphoblastic leukemia ${ }^{75}$ and Burkitt lymphoma ${ }^{76,77}$ in which it remains an accepted standard of care.

These results have been corroborated in DLBCL with the phase III GELA trial comparing CHOP-21 (cyclophosphamide, doxorubicin, vincristine and prednisolone at 21day intervals) against the intensive ACVBP regimen (doxorubicin, cyclophosphamide, vindesine, bleomycin, prednisone induction followed by sequential consolidation therapy) in patients with aggressive non-Hodgkin lymphoma and IPI $>1 .^{58}$ The ACVBP arm included four doses of intrathecal methotrexate, plus two infusions of highdose systemic methotrexate at $3000 \mathrm{mg} / \mathrm{m}^{2}$. Results of the trial were notable for significantly fewer CNS recurrences in the ACVBP arm compared to the CHOP arm (2.7\% versus $8 \% ; P=0.004)$, as well as an overall survival benefit. Greater systemic disease control with the more intensive ACVBP regimen may well account for some of the observed benefit over CHOP, but the lower rate of isolated CNS relapse suggests that CNS prophylaxis may also have played an important role. Given the lack of appreciable benefit in numerous prior studies evaluating intrathecal methotrexate alone, it is reasonable to consider that the intravenous methotrexate contributed to the reduction in the rate of CNS recurrence.

We described our retrospective experience adding systemic high-dose methotrexate to R-CHOP as CNS prophylaxis in selected high-risk patients with DLBCL. ${ }^{80}$ Patients received at least one dose of intravenous methotrexate at a dose of $3500 \mathrm{mg} / \mathrm{m}^{2}$ administered on day 15 of alternating cycles (i.e. cycles 2, 4, and 6) of R-CHOP. The population had a significant proportion of high-risk patients with IPI scores of $3-5$ in $68 \%$, elevated lactate dehydrogenase concentration in $73 \%$, more than one extranodal site of involvement in $62 \%$ of subjects, and frequent involvement of high-risk locations including the kidneys, adrenal glands, testes, bone marrow, or the epidural space. Among 65 high-risk patients, two CNS recurrences (3\%) occurred (one at 4 months and the other at 9 months). The median follow-up for the entire population was 33 months, and the 3-year progression-free survival was $76 \%$. Toxicities noted within this population included 26 patients (39\%) with creatinine elevation above the upper limit of normal, although only one patient required temporary hemodialysis and subsequently recovered renal function. Renal toxicity led to discontinuation of methotrexate in nine patients $(14 \%)$, all of whom recovered baseline renal function. In eight patients $(12 \%)$ the subsequent R-CHOP cycle had to be delayed by 1-3 weeks because of toxicity (nephrotoxicity in 4, mucositis in 2, and cytopenias in 2). Despite these adverse events, the study demonstrated that patients with normal baseline renal function could tolerate high-dose methotrexate treatment intercalated with R-CHOP therapy, and that this was associated with a lower rate of CNS relapse than may be expected based on their high-risk features at baseline. ${ }^{18}$ 
Two other retrospective studies evaluated high-dose methotrexate for CNS prophylaxis in DLBCL. In one Italian center, high-dose methotrexate (with or without intrathecal liposomal cytarabine at the discretion of the treating physician) was administered after completion of all cycles of R-CHOP for three or four cycles in patients deemed at high risk of CNS recurrence. ${ }^{66}$ These patients were then retrospectively compared to patients with highrisk features treated with no CNS prophylaxis. At a median of 60 months, $12 \%$ of patients who did not receive prophylaxis had had a CNS relapse versus $2.5 \%$ of those who received prophylaxis $(P=0.03)$, suggesting that CNS prophylaxis was beneficial. Of note, there were differences in risk factors between the two populations with more patients being defined as having high-risk disease due to advanced stage and elevated lactate dehydrogenase concentration in the group that received no prophylaxis, while high-risk anatomic locations including testis, kidney and orbit were enriched in the prophylaxed population. Such differences in patient selection complicate the interpretation of all retrospective analyses, so that conclusions can be considered suggestive but not definitive. That said, a third retrospective analysis reported concordant results with lower rates of CNS relapse in patients treated with a combination of high-dose intravenous methotrexate and intrathecal methotrexate compared to intrathecal methotrexate alone with a hazard ratio for CNS relapse at 3 years of 0.26 ( $95 \%$ confidence interval: $0.08-0.81)$ based on multivariate analysis. ${ }^{79}$

Two prospective trials have incorporated high-dose methotrexate and cytarabine, in addition to other CNSactive agents, for high-risk patients with DLBCL. A phase II trial of R-CODOX-M/IVAC (cyclophosphamide, vincristine, doxorubicin, methotrexate, ifosfamide, etoposide, and cytarabine) was performed in patients with newly diagnosed DLBCL and an IPI score of $\geq 3 .^{80}$ Among 96 patients with no CNS involvement at diagnosis, 41 had CNS-IPI scores of 2-3 (intermediate risk) and 55 had CNSIPI scores of 4-6 (high risk); the rates of CNS relapse in these groups at 2 years were $0 \%$ and $6 \%$, respectively, which are lower than might have been predicted without CNS-directed therapy, although the concomitant toxicity of these intensive regimens must be taken into account. The Nordic Lymphoma Study Group performed a phase II study in patients with high-risk DLBCL or grade 3 follicular lymphoma, with age-adjusted IPI scores of $2-3 .{ }^{23}$ Treatment consisted of six cycles of R-CHOEP-14 (RCHOP-14 plus etoposide) followed by cytarabine at 3000

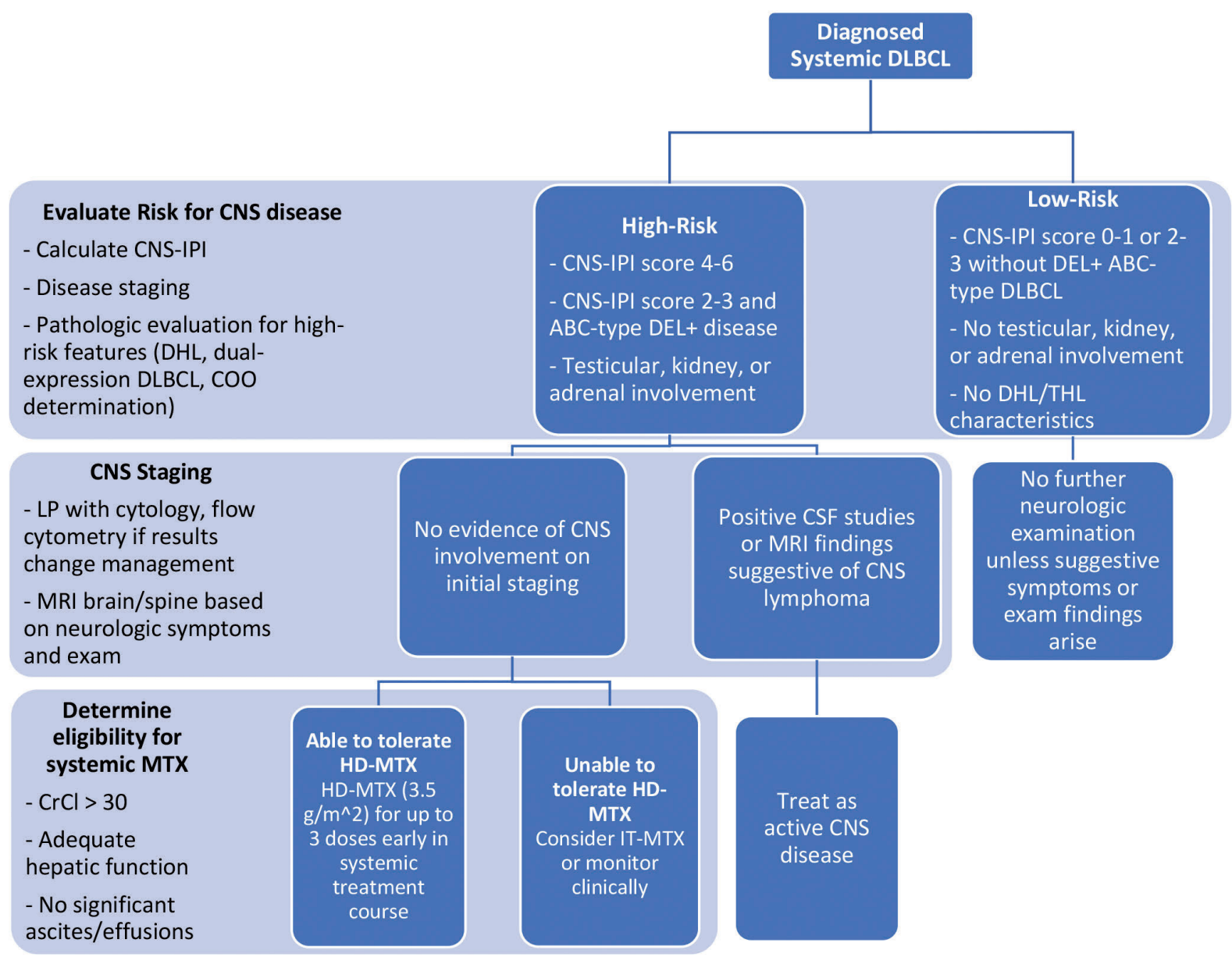

Figure 2. Suggested approach to central nervous system risk stratification and prophylaxis in newly diagnosed diffuse large B-cell lymphoma. DLBCL: diffuse large B-cell Iymphoma; CNS-IPI: Central Nervous System International Prognostic Index; DHL: double-hit lymphoma; COO: cell of origin; ABC: activated B-cell; DEL: doubleexpressing lymphoma; THL: triple-hit lymphoma; LP: Iumbar puncture; MRI: magnetic resonance imaging; CNS: central nervous system; CSF: cerebrospinal fluid; MTX: methotrexate; CrCl: creatine clearance; HD-MTX: high-dose methotrexate; IT-MTX: intrathecal methotrexate. 
$\mathrm{mg} / \mathrm{m}^{2}$ twice daily for 2 days, followed 3 weeks later by methotrexate at a dose of $3000 \mathrm{mg} / \mathrm{m}^{2}$. Among 156 patients, there were three toxicity-related deaths. The rate of CNS relapse was $4.5 \%$, and all relapses occurred within 6 months of diagnosis; this CNS recurrence rate is comparable to that in historical controls. It is interesting to note, however, that all relapses occurred very early in the disease course and the CNS-active agents were administered only following induction therapy. This supports the hypothesis that occult CNS involvement may already have been present early in the disease course, and that earlier administration of systemic methotrexate may have conferred greater benefit.

The optimal timing of CNS prophylaxis, particularly with high-dose intravenous methotrexate, remains incompletely elucidated. CNS relapse typically occurs during or within 5-6 months of induction treatment.,28 The incidence of early CNS events forms the rationale for introduction of CNS-directed therapy concurrently with systemic induction treatment, but this must be balanced with risks of toxicity due to concomitant therapy. For high-risk DLBCL patients who are appropriate candidates for highdose systemic methotrexate, we favor inclusion at a dose of $3500 \mathrm{mg} / \mathrm{m}^{2}$ on day 15 of the 21-day R-CHOP cycle for up to a total of three doses, usually administered in alternating $\mathrm{R}-\mathrm{CHOP}$ cycles. An alternative schedule is to administer three or four doses of intravenous methotrexate immediately following completion of R-CHOP, although this risks earlier CNS progression. Methotrexate can be administered at 10- to 14-day intervals when given as monotherapy following completion of R-CHOP. A recommended algorithm for risk stratification and prophylaxis is shown in Figure 2.

Safe administration of high-dose systemic methotrexate for CNS prophylaxis relies upon careful attention to the selection and supportive care of patients. This treatment should be avoided in patients with a poor performance status, and those with impaired renal function or significant effusions or ascites which may serve as reservoirs for methotrexate and prolong toxicity. In order to minimize risk of toxicity, patients are pre-treated with hydration and alkalinization, which continues after methotrexate infusion to accelerate clearance. Methotrexate at a dose of $3000-3500 \mathrm{mg} / \mathrm{m}^{2}$ is typically administered over $2-4 \mathrm{~h}$, with leucovorin rescue commencing $24 \mathrm{~h}$ after the beginning of the methotrexate infusion, and continuing every 6 $\mathrm{h}$ for 12-16 doses as the methotrexate clears. It is essential to monitor methotrexate levels along with electrolytes and renal function in order to ensure that the drug is cleared rapidly, which helps to avoid toxicity.

There are no data to support systemic prophylaxis with chemotherapeutic agents other than methotrexate at this time. Cytarabine has activity as a single agent and in combination with high-dose methotrexate in primary CNS lymphoma, but has not been validated to add benefit as prophylaxis in systemic DLBCL. ${ }^{74,81}$ Etoposide is a widely used lymphoma therapy which may attain cytotoxic concentrations in the CSF and had historically been associated with a reduced risk of CNS recurrence, raising the prospect of benefit in the prophylactic setting. ${ }^{8,82}$ Subsequent studies of etoposide with rituximab-containing therapy in the modern era, however, found no significant benefit in reducing CNS risk, so etoposide also cannot be recommended as a component of prophylactic therapy. ${ }^{18,83}$
Two novel agents, ibrutinib and lenalidomide, have demonstrated activity in relapsed DLBCL, particularly in ABC-like DLBCL which characterizes most cases of primary and secondary CNS lymphoma. Additionally, ibrutinib appears especially promising in ABC-like DLBCL harboring both $M Y D 88$ and $C D 79 B$ mutations, a mutational pattern commonly observed in primary CNS DLBCL. ${ }^{84.86}$ Given this biological rationale, ibrutinib and lenalidomide have both been preliminarily investigated in primary CNS DLBCL in which they have demonstrated the ability to cross the blood-brain barrier and induce remissions. . $^{8788}$ Based on these findings, BTK inhibitors and lenalidomide warrant evaluation in the therapy of secondary CNS lymphoma as well. Whether incorporation of one or both of these novel agents into upfront therapy in high-risk patients will reduce the risk of CNS relapse remains unknown, but will likely be elucidated by randomized trials adding these agents to R-CHOP in ABC-like DLBCL, which have been completed and await reporting. Two phase II trials in which lenalidomide was added to RCHOP included 136 patients with CNS-IPI intermediateand high-risk scores present in $71.3 \%$ and $18.4 \%$, respectively. ${ }^{89}$ Prophylactic intrathecal methotrexate was employed in only $14 \%$ of patients. At a median follow-up of 48 months, only one of the 136 patients had experienced a CNS relapse, which is a promising early result. In addition to evaluating these agents in patients with active CNS DLBCL, studies will be helpful in determining the potential benefit of these agents in preventing CNS relapse, and in determining which patients could derive the most benefit from these novel therapies.

The advent of immune checkpoint inhibitors in the treatment of solid tumors and Hodgkin lymphoma ${ }^{90}$ has garnered interest in their use for DLBCL. While PD-L1 expression is uncommon in systemic DLBCL, ${ }^{91}$ higher rates of PD-L1 expression have been noted in primary mediastinal large $\mathrm{B}$-cell lymphoma, ${ }^{92}$ primary CNS DLBCL, and primary testicular DLBCL. ${ }^{35} \mathrm{~A}$ small case series including four patients with relapsed/refractory primary CNS DLBCL and one patient with CNS relapse of primary testicular DLBCL showed clinical and radiographic responses in all patients treated with the PD-1 inhibitor nivolumab..$^{93}$ These early data warrant further investigation to determine whether select subsets of high-risk DLBCL patients may benefit from immune checkpoint inhibition to reduce the risk of CNS relapse.

\section{Conclusions}

In patients with DLBCL, relapse within the CNS remains a rare but devastating complication. There are significant limitations to determining the optimal methods of risk stratification and prophylaxis against CNS relapse, including the infrequency of the event, heterogeneity of existing literature, and inability to enroll sufficient numbers of patients in appropriately powered clinical trials with the primary outcome of CNS relapse. As a result, recommendations and guidelines remain largely empiric in nature.

Based on the available data and clinical experience, the optimal approach is to first consider patient- and diseasespecific risk factors and identify patients at highest risk for CNS relapse. Proper risk stratification should include calculation of patients' CNS-IPI score, consideration of extranodal sites of disease, and identification of disease-specific 
biological factors including double- or triple-hit translocations and double-expresser status in concert with determination of the cell of origin. Baseline CNS evaluation may include CSF studies with cytology and flow cytometry based on patient-specific risk factors, and imaging of the neuroaxis if neurological signs or symptoms are present. For those patients at high risk of CNS relapse, prophylactic therapy should be considered. Our preference is to employ systemic methotrexate as first-line CNS prophylaxis if the patient is an appropriate candidate, with intrathecal methotrexate reserved for high-risk patients who are ineligible for systemic methotrexate because of renal dysfunction or other comorbidities. Early administration of CNS prophylaxis, during the course of initial therapy, may treat occult CNS disease and prevent early treatment failure. Finally, new targeted therapies and immune-modulating agents may provide novel opportunities for the treatment and prevention of CNS relapse in the future. Inclusion of patients with secondary CNS lymphoma in clinical trials of promising agents would significantly accelerate the rate of progress in this currently unmet medical need.

\section{References}

1. Morton LM, Wang SS, Devesa SS, et al. Lymphoma incidence patterns by WHO subtype in the United States, 1992-2001. Blood. 2006;107(1):265-276.

2. Swerdlow SH, Campo E, Pileri SA, et al. The 2016 revision of the World Health Organization classification of lymphoid neoplasms. Blood. 2016;127(20):2375-2390.

3. Ziepert M, Hasenclever D, Kuhnt E, et al. Standard International prognostic index remains a valid predictor of outcome for patients with aggressive CD20+ B-cell lymphoma in the rituximab era. J Clin Oncol. 2010;28(14):2373-2380.

4. Smith A, Crouch S, Howell D, et al. Impact of age and socioeconomic status on treatment and survival from aggressive lymphoma: a UK population-based study of diffuse large B-cell lymphoma. Cancer Epidemiol. 2015;39(6):1103-1112.

5. Sehn LH, Berry B, Chhanabhai M, et al. The revised International Prognostic Index (RIPI) is a better predictor of outcome than the standard IPI for patients with diffuse large Bcell lymphoma treated with R-CHOP. Blood. 2007;109(5):1857-1861.

6. Ghose A, Elias HK, Guha G, et al. Influence of rituximab on central nervous system relapse in diffuse large B-cell lymphoma and role of prophylaxis--a systematic review of prospective studies. Clin Lymphoma Myeloma Leuk. 2015;15(8):451-457.

7. Zhang J, Chen B, Xu X. Impact of rituximab on incidence of and risk factors for central nervous system relapse in patients with diffuse large B-cell lymphoma: a systematic review and meta-analysis. Leuk Lymphoma. 2014:55(3):509-514.

8. Boehme V, Zeynalova S, Kloess M, et al. Incidence and risk factors of central nervous system recurrence in aggressive lymphoma-a survey of 1693 patients treated in protocols of the German High-Grade NonHodgkin's Lymphoma Study Group (DSHNHL). Ann Oncol. 2007;18(1):149-157.

9. Bjorkholm M, Hagberg $\mathrm{H}$, Holte $\mathrm{H}$, et al. Central nervous system occurrence in elderly patients with aggressive lymphoma and a long-term follow-up. Ann Oncol. 2007;18(6):1085-1089.

10. Bernstein SH, Unger JM, Leblanc M, et al. Natural history of CNS relapse in patients with aggressive non-Hodgkin's lymphoma: a 20-year follow-up analysis of SWOG 8516 -- the Southwest Oncology Group. J Clin Oncol. 2009:27(1):114-119.

11. Wilson WH, Bromberg JE, Stetler-Stevenson $\mathrm{M}$, et al. Detection and outcome of occult leptomeningeal disease in diffuse large B-cell lymphoma and Burkitt lymphoma. Haematologica, 2014;99(7):1228-1235.

12. Hegde U, Filie A, Little RF, et al. High incidence of occult leptomeningeal disease detected by flow cytometry in newly diagnosed aggressive B-cell lymphomas at risk for central nervous system involvement: the role of flow cytometry versus cytology. Blood. 2005;105(2):496-502.

13. Benevolo G, Stacchini A, Spina M, et al. Final results of a multicenter trial addressing role of CSF flow cytometric analysis in NHL patients at high risk for CNS dissemination. Blood. 2012;120(16):3222-3228.

14. Alvarez R, Dupuis J, Plonquet A, et al. Clinical relevance of flow cytometric immunophenotyping of the cerebrospinal fluid in patients with diffuse large B-cell lymphoma. Ann Oncol. 2012;23(5):12741279.

15. Sancho JM, Orfao A, Quijano S, et al. Clinical significance of occult cerebrospinal fluid involvement assessed by flow cytometry in non-Hodgkin's lymphoma patients at high risk of central nervous system disease in the rituximab era. Eur J Haematol. 2010;85(4):321-328.

16. Boehme V, Schmitz N, Zeynalova S, et al. CNS events in elderly patients with aggressive lymphoma treated with modern chemotherapy (CHOP-14) with or without rituximab: an analysis of patients treated in the RICOVER-60 trial of the German HighGrade Non-Hodgkin Lymphoma Study Group (DSHNHL). Blood. 2009;113(17): 3896-3902.

17. El-Galaly TC, Villa D, Michaelsen TY, et al. The number of extranodal sites assessed by $\mathrm{PET} / \mathrm{CT}$ scan is a powerful predictor of CNS relapse for patients with diffuse large B-cell lymphoma: An international multicenter study of 1532 patients treated with chemoimmunotherapy. Eur J Cancer. 2017;75:195-203.

18. Schmitz N, Zeynalova S, Nickelsen M, et al. CNS International Prognostic Index: a risk model for CNS relapse in patients with diffuse large B-cell lymphoma treated with RCHOP. J Clin Oncol. 2016;34(26):3150-3156.

19. Gleeson M, Counsell N, Cunningham D, et al. Central nervous system relapse of diffuse large B-cell lymphoma in the rituximab era: results of the UK NCRI R-CHOP-14 versus 21 trial. Ann Oncol. 2017;28(10):2511-2516.

20. Guirguis HR, Cheung MC, Mahrous M, et al. Impact of central nervous system (CNS) prophylaxis on the incidence and risk factors for CNS relapse in patients with diffuse large B-cell lymphoma treated in the rituximab era: a single centre experience and review of the literature. $\mathrm{Br} \mathrm{J}$ Haematol. 2012;159(1):39-49.
21. Mitrovic Z, Bast M, Bierman PJ, et al. The addition of rituximab reduces the incidence of secondary central nervous system involvement in patients with diffuse large Bcell lymphoma. Br J Haematol. 2012;157 (3):401-403

22. Kumar A, Vanderplas A, LaCasce AS, et al. Lack of benefit of central nervous system prophylaxis for diffuse large B-cell lymphoma in the rituximab era: findings from a large national database. Cancer. 2012;118 (11):2944-2951.

23. Holte H, Leppa S, Bjorkholm M, et al. Dosedensified chemoimmunotherapy followed by systemic central nervous system prophylaxis for younger high-risk diffuse large Bcell/follicular grade 3 lymphoma patients: results of a phase II Nordic Lymphoma Group study. Ann Oncol. 2013;24(5):13851392.

24. Uni M, Kagoya Y, Nannya Y, et al. Central nervous system relapse in patients with diffuse large B-cell lymphoma: analysis of incidence and prognostic factors. Leuk Lymphoma. 2015;56(6):1869-1871.

25. Blum KA, Lozanski G, Byrd JC. Adult Burkitt leukemia and lymphoma. Blood. 2004;104(10):3009-3020.

26. Cortes J, O'Brien SM, Pierce S, et al. The value of high-dose systemic chemotherapy and intrathecal therapy for central nervous system prophylaxis in different risk groups of adult acute lymphoblastic leukemia. Blood. 1995;86(6):2091-2097.

27. Haioun C, Besson C, Lepage E, et al. Incidence and risk factors of central nervous system relapse in histologically aggressive non-Hodgkin's lymphoma uniformly treated and receiving intrathecal central nervous system prophylaxis: a GELA study on 974 patients. Ann Oncol. 2017;11(6):685-690.

28. Hollender A, Kvaloy S, Nome O, et al. Central nervous system involvement following diagnosis of non-Hodgkin's lymphoma: a risk model. Ann Oncol. 2002;13 (7):1099-1107.

29. A predictive model for aggressive nonHodgkin's lymphoma. N Engl J Med. 1993; 329(14):987-994.

30. Tomita N, Yokoyama M, Yamamoto W, et al. The standard international prognostic index for predicting the risk of CNS involvement in DLBCL without specific prophylaxis. Leuk Lymphoma. 2018;59(1):97-104.

31. Kridel R, Telio D, Villa D, et al. Diffuse large B-cell lymphoma with testicular involvement: outcome and risk of CNS relapse in the rituximab era. Br J Haematol. 2017;176 (2):210-221.

32. Zucca E, Conconi A, Mughal TI, et al Patterns of outcome and prognostic factors in primary large-cell lymphoma of the testis 
in a survey by the International Extranodal Lymphoma Study Group. J Clin Oncol. 2003;21(1):20-27.

33. Mazloom A, Fowler N, Medeiros LJ, et al. Outcome of patients with diffuse large Bcell lymphoma of the testis by era of treatment: the M. D. Anderson Cancer Center experience. Leuk Lymphoma. 2010;51(7): 1217-1224.

34. Fonseca R, Habermann TM, Colgan JP, et al. Testicular lymphoma is associated with a high incidence of extranodal recurrence. Cancer. 2000;88(1):154-161.

35. Chapuy B, Roemer MG, Stewart C, et al. Targetable genetic features of primary testicular and primary central nervous system lymphomas. Blood. 2016;127(7):869-881.

36. Deng L, Xu-Monette ZY, Loghavi S, et al. Primary testicular diffuse large B-cell lymphoma displays distinct clinical and biological features for treatment failure in rituximab era: a report from the International PTL Consortium. Leukemia. 2016;30(2):361-372.

37. Yao Z, Deng L, Xu-Monette ZY, et al. Concordant bone marrow involvement of diffuse large B-cell lymphoma represents a distinct clinical and biological entity in the era of immunotherapy. Leukemia. 2018;32(2):353-363

38. Laskin JJ, Savage KJ, Voss N, et al. Primary paranasal sinus lymphoma: natural history and improved outcome with central nervous system chemoprophylaxis. Leuk Lymphoma. 2005;46(12):1721-1727.

39. Lee GW, Go SI, Kim SH, et al. Clinical outcome and prognosis of patients with primary sinonasal tract diffuse large B-cell lymphoma treated with rituximab-cyclophosphamide, doxorubicin, vincristine and prednisone chemotherapy: a study by the Consortium for Improving Survival of Lymphoma. Leuk Lymphoma. 2015;56(4): 1020-1026.

40. El-Galaly TC, Cheah CY, Hutchings M, et al. Uterine, but not ovarian, female reproductive organ involvement at presentation by diffuse large B-cell lymphoma is associated with poor outcomes and a high frequency of secondary CNS involvement. Br J Haematol. 2016;175(5):876-883.

41. Aviv A, Tadmor T, Polliack A. Primary diffuse large B-cell lymphoma of the breast: looking at pathogenesis, clinical issues and therapeutic options. Ann Oncol. 2013;24(9): 2236-2244.

42. Murawski N, Held G, Ziepert M, et al. The role of radiotherapy and intrathecal $\mathrm{CNS}$ prophylaxis in extralymphatic craniofacial aggressive B-cell lymphomas. Blood. 2014;124(5):720-728

43. Lehners N, Kramer I, Schwarzbich MA, et al. Analysis of clinical characteristics and outcome of patients with previously untreated diffuse large B-cell lymphoma and renal involvement in the rituximab era. Leuk Lymphoma. 2016;57(11):2619-2625.

44. Tomita N, Yokoyama M, Yamamoto W, et al. Central nervous system event in patients with diffuse large B-cell lymphoma in the rituximab era. Cancer Sci. 2012;103(2):245251.

45. Savage KJ, Johnson NA, Ben-Neriah S, et al. MYC gene rearrangements are associated with a poor prognosis in diffuse large B-cell lymphoma patients treated with $\mathrm{R}-\mathrm{CHOP}$ chemotherapy. Blood. 2009;114(17):35333537

46. Barrans S, Crouch S, Smith A, et al. Rearrangement of MYC is associated with poor prognosis in patients with diffuse large
B-cell lymphoma treated in the era of rituximab. J Clin Oncol. 2010;28(20):3360-3365.

47. Petrich AM, Gandhi M, Jovanovic B, et al Impact of induction regimen and stem cell transplantation on outcomes in double-hit lymphoma: a multicenter retrospective analysis. Blood. 2014;124(15):2354-2361

48. Oki Y, Noorani M, Lin P, et al. Double hit ymphoma: the MD Anderson Cancer Center clinical experience. Br J Haematol. 2014;166(6):891-901.

49. Kanungo A, Medeiros LJ, Abruzzo LV, et al. Lymphoid neoplasms associated with concurrent $\mathrm{t}(14 ; 18)$ and 8q24/c-MYC translocation generally have a poor prognosis. Mod Pathol. 2006;19(1):25-33.

50. Le Gouill S, Talmant P, Touzeau C, et al. The clinical presentation and prognosis of diffuse large B-cell lymphoma with $\mathrm{t}(14 ; 18)$ and 8q24/c-MYC rearrangement. Haematologica. 2007;92(10):1335-1342.

51. Savage KJ, Slack GW, Mottok A, et al. Impact of dual expression of MYC and BCL2 by immunohistochemistry on the risk of CNS relapse in DLBCL. Blood. 2016;127(18):2182-2188.

52. Hu S, Xu-Monette ZY, Tzankov A, et al MYC/BCL2 protein coexpression contributes to the inferior survival of activated B-cell subtype of diffuse large B-cell lymphoma and demonstrates high-risk gene expression signatures: a report from The International DLBCL Rituximab-CHOP Consortium Program. Blood. 2013;121(20): 4021-4031.

53. Song YS, Lee WW, Lee IS, et al. Prediction of Central nervous system relapse of diffuse large B-cell lymphoma using pretherapeuti [18F]2-fluoro-2-deoxyglucose (FDG) positron emission tomography/computed tomography. Medicine. 2015;94(44):e1978.

54. Lemma SA, Pasanen AK, Haapasaari KM, et al. Similar chemokine receptor profiles in lymphomas with central nervous system involvement - possible biomarkers for patient selection for central nervous system prophylaxis, a retrospective study. Eur J Haematol. 2016;96(5):492-501.

55. Lemma SA, Kuusisto M, Haapasaari KM, et al. Integrin alpha 10, CD44, PTEN, cadherin11 and lactoferrin expressions are potential biomarkers for selecting patients in need of central nervous system prophylaxis in diffuse large B-cell lymphoma. Carcinogenesis. 2017;38(8):812-820.

56. Glass JP, Melamed M, Chernik NL, et al. Malignant cells in cerebrospinal fluid (CSF) the meaning of a positive CSF cytology. Neurology. 1979;29(10):1369-1375.

57. van Besien K, Ha CS, Murphy S, et al. Risk factors, treatment, and outcome of centra nervous system recurrence in adults with intermediate-grade and immunoblastic lymphoma. Blood. 1998;91(4):1178-1184.

58. Tilly $\mathrm{H}$, Lepage E, Coiffier $\mathrm{B}$, et al. Intensive conventional chemotherapy (ACVBP regimen) compared with standard $\mathrm{CHOP}$ for poor-prognosis aggressive non-Hodgkin lymphoma. Blood. 2003;102(13):4284-4289.

59. Baraniskin A, Kuhnhenn J, Schlegel U, et al Identification of microRNAs in the cere brospinal fluid as marker for primary diffuse large B-cell lymphoma of the central nervous system. Blood. 2011;117(11):3140-3146.

60. Baraniskin A, Zaslavska E, NopelDunnebacke $S$, et al. Circulating U2 small nuclear RNA fragments as a novel diagnostic biomarker for primary central nervous system lymphoma. Neuro Oncol. 2016;18(3): 361-367.
61. Martinez-Ricarte F, Mayor R, Martinez-Saez $\mathrm{E}$, et al. Molecular diagnosis of diffuse gliomas through sequencing of cell-free circulating tumor DNA from cerebrospinal fluid. Clin Cancer Res. 2018;24(12):2812 2819.

62. Pentsova EI, Shah RH, Tang J, et al Evaluating cancer of the central nervous system through next-generation sequencing of cerebrospinal fluid. J Clin Oncol. 2016;34 (20):2404-2415

63. Hutchings $M$, Ladetto $M$, Buske $C$, et al ESMO Consensus Conference on malignant lymphoma: management of 'ultra-high-risk' patients. Ann Oncol. 2018;29(8):1687-1700.

64. Schmitz N, Zeynalova S, Glass B, et al. CNS disease in younger patients with aggressive B-cell lymphoma: an analysis of patients treated on the Mabthera International Trial and trials of the German High-Grade NonHodgkin Lymphoma Study Group. Ann Oncol. 2012;23(5):1267-1273.

65. Tomita N, Takasaki H, Ishiyama Y, et al. Intrathecal methotrexate prophylaxis and central nervous system relapse in patients with diffuse large B-cell lymphoma following rituximab plus cyclophosphamide, doxorubicin, vincristine and prednisone. Leuk Lymphoma. 2015;56(3):725-729.

66. Ferreri AJ, Bruno-Ventre M, Donadoni G, et al. Risk-tailored CNS prophylaxis in a mono-institutional series of 200 patients with diffuse large B-cell lymphoma treated in the rituximab era. Br J Haematol. 2015;168 (5):654-662.

67. Tai WM, Chung J, Tang PL, et al. Central nervous system (CNS) relapse in diffuse large B cell lymphoma (DLBCL): pre- and post-rituximab. Ann Hematol. 2011;90(7): 809-818.

68. Shapiro WR, Young DF, Mehta BM Methotrexate: distribution in cerebrospinal fluid after intravenous, ventricular and lumbar injections. N Engl J Med. 1975;293(4): 161-166.

69. Chamberlain MC. Radioisotope CSF flow studies in leptomeningeal metastases. J Neurooncol. 1998;38(2-3):135-140.

70. Blasberg RG, Patlak C, Fenstermacher JD Intrathecal chemotherapy: brain tissue profiles after ventriculocisternal perfusion. I Pharmacol Exp Ther. 1975;195(1):73-83.

71. Dunleavy K, Pittaluga S, Shovlin M, et al Low-intensity therapy in adults with Burkitt's lymphoma. $N$ Engl J Med. 2013;369(20):1915-1925.

72. Niemann A, Muhlisch J, Fruhwald MC, et al Therapeutic drug monitoring of methotrexate in cerebrospinal fluid after systemic high-dose infusion in children: can the burden of intrathecal methotrexate be reduced? Ther Drug Monit. 2010;32(4):467-475.

73. Ferreri AJ, Reni M, Pasini F, et al. A multicenter study of treatment of primary CNS lymphoma. Neurology. 2002;58(10):15131520 .

74. Ferreri AJ, Reni M, Foppoli M, et al. Highdose cytarabine plus high-dose methotrexate versus high-dose methotrexate alone in patients with primary CNS lymphoma: a randomised phase 2 trial. Lancet. 2009;374 (9700):1512-1520.

75. Pui CH, Campana D, Pei D, et al. Treating childhood acute lymphoblastic leukemia without cranial irradiation. $\mathrm{N}$ Engl J Med. 2009;360(26):2730-2741.

76. Magrath I, Adde M, Shad A, et al. Adults and children with small non-cleaved-cel lymphoma have a similar excellent outcome when treated with the same chemotherapy 
regimen. J Clin Oncol. 1996;14(3):925-934

77. Rizzieri DA, Johnson JL, Byrd JC, et al. Improved efficacy using rituximab and brief duration, high intensity chemotherapy with filgrastim support for Burkitt or aggressive lymphomas: cancer and Leukemia Group B study 10 002. Br J Haematol. 2014;165(1): 102-111.

78. Abramson JS, Hellmann M, Barnes JA, et al. Intravenous methotrexate as central nervous system (CNS) prophylaxis is associated with a low risk of CNS recurrence in high-risk patients with diffuse large B-cell lymphoma. Cancer. 2010;116(18):4283-4290.

79. Cheah CY, Herbert KE, O'Rourke K, et al. A multicentre retrospective comparison of central nervous system prophylaxis strategies among patients with high-risk diffuse large B-cell lymphoma. Br J Cancer. 2014;111(6):1072-1079.

80. Phillips E, Kirkwood A, Lawrie A, et al. Low rates of CNS relapse in high risk DLBCL patients treated with R-CODOX-M and RIVAC: results from a phase 2 UK NCRI/bloodwise trial. Blood. 2016;128 (22):1855.

81. Herzig RH, Hines JD, Herzig GP, et al. Cerebellar toxicity with high-dose cytosine arabinoside J Clin Oncol. 1987:5(6):927-932.

82. Relling MV, Mahmoud $\mathrm{HH}$, Pui $\mathrm{CH}$, et al. Etoposide achieves potentially cytotoxic concentrations in CSF of children with acute lymphoblastic leukemia. J Clin Oncol. 1996;14(2):399-404.

83. Malecek MK, Petrich AM, Rozell S, et al Frequency, risk factors, and outcomes of central nervous system relapse in lymphoma patients treated with dose-adjusted EPOCH plus rituximab. Am J Hematol. 2017;92 (11):1156-1162.

84. Ngo VN, Young RM, Schmitz R, et al Oncogenically active MYD88 mutations in human lymphoma. Nature. 2011;470 (7332):115-119.

85. Davis RE, Ngo VN, Lenz G, et al. Chronic active $\mathrm{B}$ cell receptor signaling in diffuse large B cell lymphoma. Nature. 2010;463 (7277):88-92.

86. Lenz G, Davis RE, Ngo VN, et al. Oncogenic CARD11 mutations in human diffuse large B cell lymphoma. Science. 2008;31 (5870):1676-1679.

87. Lionakis MS, Dunleavy K, Roschewski M, et al. Inhibition of $B$ cell receptor signaling by ibrutinib in primary CNS lymphoma. Cancer Cell. 2017;31(6):833-843.e5.
88. Houillier C, Choquet S, Touitou V, et al. Lenalidomide monotherapy as salvage treatment for recurrent primary CNS lymphoma. Neurology. 2015;84(3):325-326.

89. Ayed AO, Chiappella A, Pederson L, et al CNS relapse in patients with DLBCL treated with lenalidomide plus R-CHOP (R2CHOP): analysis from two phase 2 studies. Blood Cancer J. 2018;8(7):63.

90. Ansell SM, Lesokhin AM, Borrello I, et al. PD-1 blockade with nivolumab in relapsed or refractory Hodgkin's lymphoma. N Engl J Med. 2015;372(4):311-319.

91. Kiyasu J, Miyoshi H, Hirata A, et al Expression of programmed cell death ligand 1 is associated with poor overall survival in patients with diffuse large B-cell lymphoma. Blood. 2015;126(19):2193-2201.

92. Twa DD, Chan FC, Ben-Neriah S, et al. Genomic rearrangements involving programmed death ligands are recurrent in primary mediastinal large B-cell lymphoma. Blood. 2014;123(13):2062-2065.

93. Nayak L, Iwamoto FM, LaCasce A, et al. PD-1 blockade with nivolumab in relapsed/refractory primary central nervous system and testicular lymphoma. Blood. 2017;129(23):3071-3073. 